\title{
Asymmetric interference between components of suprathreshold compound gratings
}

\author{
H. C. HUGHES \\ Dartmouth College, Hanover, New Hampshire
}

\begin{abstract}
Observers were presented with compound gratings consisting of a high- $(5.0 \mathrm{cycles} / \mathrm{deg}$, cpd) and a low-frequency $(0.5 \mathrm{cpd})$ sinusoid. The orientation of each component was independent and varied randomly between horizontal and vertical. The observers were instructed to signal the orientation of one component (the target) and ignore the other. The dependent measure was the observers' choice reaction time (CRT) to signal the orientation of the target grating. CRTs to lowfrequency targets were similar whether or not the high-frequency component was present, but CRTs to high-frequency targets were slowed by the presence of the low-frequency component. This asymmetric interference was obtained when the contrasts of the individual components were adjusted so as to produce equivalent CRTs when each was presented alone. Reducing the lowfrequency contrast below the level needed to match the CRTs generated less interference, and in some cases generated asymmetric interference favoring the high-frequency component. The magnitude of low-frequency interference was also reduced when the stimulus onsets were gradual rather than abrupt. CRTs to compounds in which the component orientations were always the same but varied randomly across trials produced little evidence of a redundancy gain, and the obtained redundancy effect was less than that predicted from a simple model of suprathreshold probability summation between independent frequency and orientation-specific channels. The results are considered in terms of inhibitory interactions between transient and sustained channels.
\end{abstract}

There is considerable evidence that the human visual system contains mechanisms ("channels") which respond to a restricted range of spatial frequencies (e.g., Campbell \& Robson, 1968; Sachs, Nachmias, \& Robson, 1971). Moreover, high-spatial-frequency channels are generally thought to respond best to low temporal frequencies (i.e., sustained channels), whereas low-spatialfrequency channels respond best at high temporal frequencies (i.e., transient channels). Thus, these psychophysically defined channels are often regarded as spatiotemporal bandpass filters (see Kelly \& Burbeck, 1984, for a review).

Results from adaptation (e.g., Blakemore \& Campbell, 1969; Pantle \& Sekuler, 1968), masking (e.g., Stromeyer \& Julesz, 1972), and subthreshold summation experiments (Graham \& Nachmias, 1971; Sachs et al., 1971) indicate that gratings in frequency ratios greater than 3:1 are detected by independent frequency-specific mechanisms. However, there are also data which suggest the existence of inhibitory interactions between frequency-specific channels. Henning, Hertz, and Broadbent (1975) have reported masking effects between widely separated spatial frequencies that have certain similarities to auditory pitch perception. The results of several adaptation studies have suggested the possibility of inhibitory interactions (e.g.,

This research was supported by a faculty research award from Dartmouth College. I wish to thank Catherine P. Cramer, George S. Borszcz, and Catherine Ruvolo for their generous service as observers, and J. Nachmias and B. Breitmeyer for their helpful comments. Address all correspondence and requests for reprints to H. C. Hughes, Department of Psychology, Dartmouth College, Hanover, NH 03755.
DeValois, 1977; Nachmias, Sansbury, Vasilev, \& Weber, 1973; Tolhurst, 1972; Tolhurst \& Barfield, 1978), and several masking experiments have shown asymmetric masking favoring the higher spatial frequencies (Nachmias \& Weber, 1975; Stromeyer \& Klein, 1974). In contrast, backward masking (Breitmeyer, 1978; Breitmeyer \& Ganz, 1976) and the periphery effect (Breitmeyer, Valberg, Kertenbach, \& Neumeyer, 1980; Valberg \& Breitmeyer, 1980) have both been interpreted as reflecting inhibition of sustained channels by transient channels. Inhibition of sustained channels by transient channels would presumably favor the processing of low spatial frequencies, since, as mentioned earlier, transient channels are thought to respond best to low-frequency stimuli (Breitmeyer, 1975; Burbeck \& Kelly, 1981; Harwerth \& Levi, 1978; Kelly \& Burbeck, 1984; Legge, 1978; Tolhurst, 1973, 1975a, 1975b).

The results of several physiological studies can also be interpreted as revealing transient-on-sustained channel inhibition in the lateral geniculate nucleus (LGNd: e.g., Dubin \& Cleland, 1977; Hoffman, Stone, \& Sherman, 1972; Levick, Cleland, \& Dubin, 1972; Noda, 1975; Singer \& Bedworth, 1973; Singer, Poppel, \& Creutzfeldt, 1972; see Lennie, 1980 , for a review). Potentially relevant for the present experiments are the reports of "crossorientation inhibition" in cortical cells (Morrone, Burr, \& Maffei, 1982; Petrov, Pigarev, \& Zenkin, 1980). These experiments have shown that cellular responses to optimal grating patterns can be strongly inhibited by the addition of a second grating (or band-limited noise) that differs widely in orientation and/or spatial frequency from the 
cell's optimal stimulus parameters. The cells do not respond to these inhibiting stimuli when presented alone.

The present paper describes a psychophysical effect that may relate to this cross-orientation inhibition. The experiments undertaken measured choice reaction times to signal the orientation (horizontal or vertical) of a sine-wave grating (the target) in the presence of a second grating (which I tentatively call the mask). The experiments examined the interference that the mask produces on the speed of target orientation judgments.

The first experiment evaluated the patterns of interference between high- and low-frequency stimuli when the patterns were presented briefly $(100 \mathrm{msec})$ with abrupt onsets and offsets. The second experiment examined the same issue using the same spatial frequencies but with gradual pattern onsets, a procedure that should confer a processing advantage to the sustained mechanisms. The results of these experiments were consistent with the hypothesis that the low-frequency grating interferes with the processing of the high-frequency grating, but that the high-frequency grating interferes very little with the processing of the low-frequency stimulus. The third experiment searched for a summation effect between orientationally redundant (i.e., both components in the same orientation) high- and low-frequency sinusoids. The underlying assumption here was that, if the channels were independent, redundant stimuli should produce facilitation of the CRTs for orientation. This was because the output of either the high- or low-frequency mechanism would be sufficient to generate the response, and if the detection times of each channel were independent random variables, the detection times for monitoring two channels should be faster than those obtained for either channel alone. Since the obtained CRTs to the redundant compounds failed to reach the levels predicted by a simple simulation of probability summation of the detection times between independent channels, the results of the third experiment are interpreted as being consistent with the hypothesis that the channels may not be independent at suprathreshold contrasts.

\section{EXPERIMENT 1}

\section{Method}

Four observers were used throughout this investigation. Each observer was emmetropic or optically corrected. All were naive except the author (H.C.H.).

Stimuli. The stimuli were displayed on a Tektronix 5110 CRT monitor (5A15N x and $y$ amplifiers, P31 phosphor). The images were sine-wave gratings which appeared through a circular aperture, $9.0^{\circ}$ in diameter. The observers viewed the gratings binocularly using natural pupils from a distance of $57 \mathrm{~cm}$. A chinrest minimized head movements. An $\times\left(0.5^{\circ}\right)$ appeared at the center of the aperture to provide a fixation point, and the observers were instructed to maintain the $X$ in good focus throughout each session.

Sine-wave gratings of two different spatial frequencies were used: a high-frequency grating of 5.0 cycles/degree (cpd) and a lowfrequency grating of $0.5 \mathrm{cpd}$. These gratings could appear in either the horizontal or vertical orientation, and could appear alone or in compound (alternated at $100 \mathrm{~Hz}$ ). The patterns were generated using an Innisfree CRT image generator (Innisfree, Cambridge, MA), which was interfaced with a computer. The percentage contrast $[100(\max$ luminance $-\min$ luminance/max luminance $+\min$ luminance)] was controlled by two matched digital-to-analog converters (one for each spatial frequency). The mean luminance of the screen was set to $0.6 \mathrm{~cd} / \mathrm{m}^{2}$. Under these conditions, the contrast of each component grating could be varied from $30 \%$ to less than $0.5 \%$. The compound gratings were produced by using the frame interleaf module of the image generator, which alternates between the two components at a rate of $100 \mathrm{~Hz}$. Single gratings were produced in the same manner, except that the contrast of the unused component was set to zero.

The observers' task was to respond to the orientation (either horizontal or vertical) of the target grating by depressing one of two microswitches as quickly as possible. A warning tone $(1000 \mathrm{~Hz}$, $250 \mathrm{msec}$ ) preceded each pattern onset by $500 \mathrm{msec}$. Two of the observers signaled horizontal with the right hand and vertical with the left hand; this was reversed for the other two observers. The observers were instructed to respond to the target as quickly and accurately as possible. Response latencies were obtained by a clockdriven interrupt routine and were accurate to the nearest millisecond.

Procedure. Prior to data acquisition, each observer was given extensive practice $(1,000-2,000$ trials) on the orientation judgment task using single gratings at both spatial frequencies (5.0 and $0.5 \mathrm{cpd}$ ). Trials were run in blocks of 100 (approximately $10 \mathrm{~min}$ per block). About $400-500$ trials were given each day until CRTs appeared to have reached asymptotic levels. At this point, formal data acquisition began.

CRTs were obtained to both high- and low-frequency gratings from all 4 observers. CRTs to the simple gratings were compared with RTs to the same grating presented in compound. In the compound condition, the observers were told which component was the target, and they were required to signal its orientation (by pressing the appropriate response key) and ignore the masker. The orientations of each component were orthogonally combined to produce four different patterns, which were always presented in equal frequency in a randomized sequence of 100 trials. When the two components were presented in the same orientation, they always appeared in sine phase. Two of the observers (G.S.B. and C.P.C.) responded to low-frequency targets first; the other two (H.C.H. and C.M.R. responded to the high frequency first. In the situations in which contrast was a variable, the particular contrast values were presented in randomized blocks of 100 trials across observers. In addition, the compound and simple grating conditions were randomized (in blocks of 100 trials), although each daily session began with 100 presentations of the spatial frequency that served as the target for that particular session.

\section{Results}

Errors. On each trial, an observer had to determine the orientation of the target grating and depress the appropriate response switch. Trials in which the observer depressed the wrong switch were excluded from the formal analysis of response times, but the error rates were analyzed separately.

The average rate of incorrect responses over all three experiments was $10.25 \%$. In Experiment 1 , the 4 observers generated an average of $12.0 \%$ errors on highfrequency targets and $10.2 \%$ on low-frequency targets. The corresponding figures for Experiment 2 were $7.8 \%$ and $11.2 \%$ for the high- and low-frequency targets, respectively.

Table 1 provides a breakdown of the error rates obtained, for high- and low-frequency targets in Experi- 
Table 1

Error Rates in Experiments 1 and 2, Averaged Across All Low-Frequency Contrasts

\begin{tabular}{ccccc}
\hline & & & \multicolumn{2}{c}{ Compound Gratings } \\
\cline { 3 - 5 } Experiment & $\begin{array}{c}\text { Target Frequency } \\
\text { (cpd) }\end{array}$ & $\begin{array}{c}\text { Single } \\
\text { Grating }\end{array}$ & $\begin{array}{c}\text { Same } \\
\text { Orientation }\end{array}$ & $\begin{array}{c}\text { Orthogonal } \\
\text { Orientations }\end{array}$ \\
\hline \multirow{2}{*}{1} & 0.5 & 10.25 & 7.4 & 12.9 \\
& 5.0 & 10.6 & 4.6 & 20.8 \\
2 & 0.5 & 7.6 & 9.1 & 16.8 \\
& 5.0 & 5.5 & 6.1 & 11.85 \\
\hline
\end{tabular}

ments 1 and 2, using simple and compound gratings. The errors on compound gratings are illustrated for the cases in which the irrelevant component was in the same orientation and in the orthogonal orientation, relative to the target orientation. It is clear that the greatest number of errors occurred when target and mask were presented in orthogonal orientations, and this may reflect a tendency to respond to the nontarget gratings. If so, this tendency

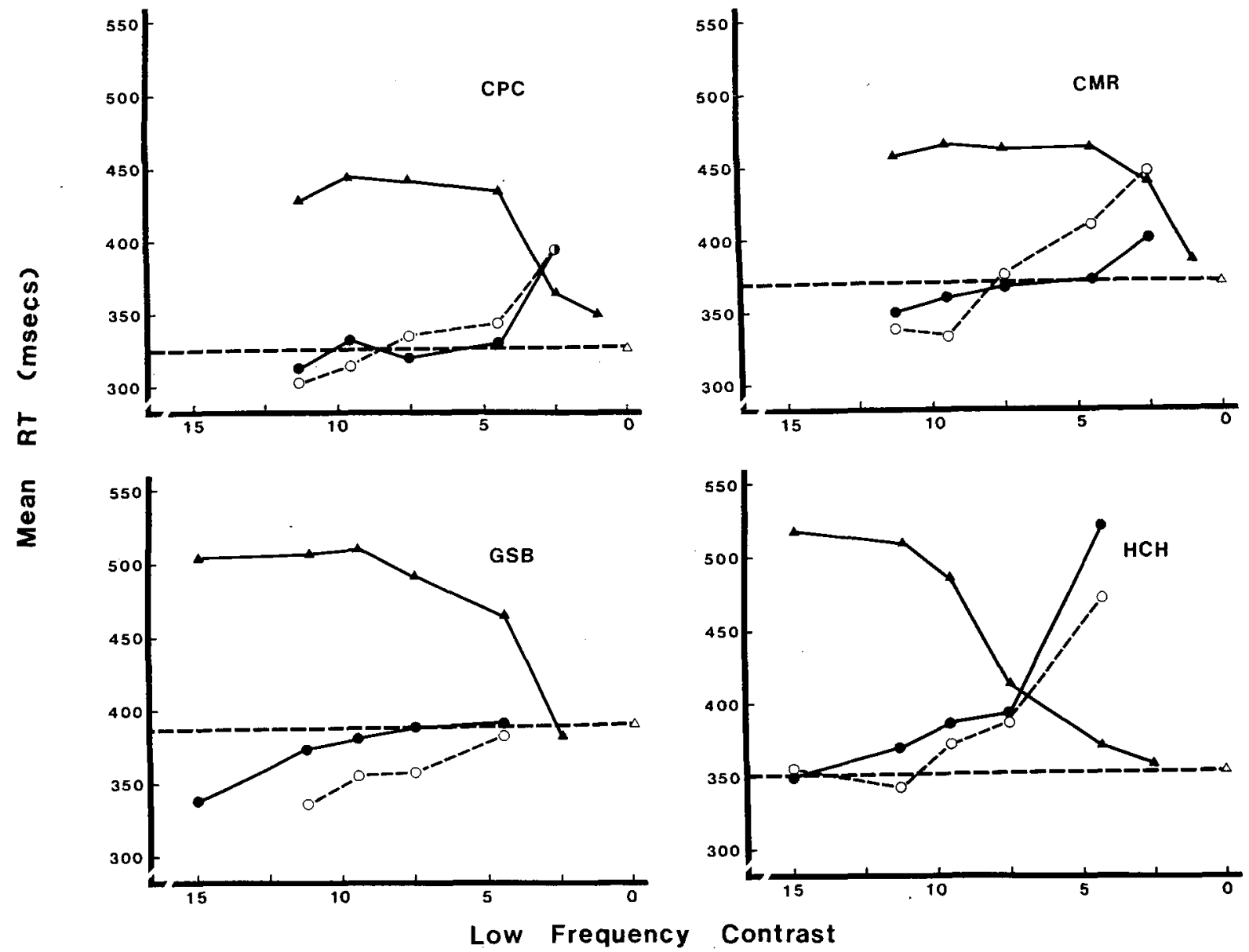

is clearly strongest, in Experiment 1, when the highfrequency grating was designated as the target (i.e., a greater tendency to respond to the lower spatial frequency). In Experiment 2, gratings in orthogonal orientations again produced the most errors, but in that case more errors were made when the responses were to the low-frequency gratings $(16.8 \%)$ than when they were to the high-frequency grating $(11.85 \%)$. The fact that, between the two experiments, a drop in error rates occurred for high-frequency targets presented with an orthogonal mask suggests less interference from the low-frequency masker in the second experiment. This suggestion finds support in the RT data reported below. Otherwise, there is little evidence of covariation between the error rates and the associated response times.

Reactions to simple gratings. The open circles in Figure 1 illustrate the relationship between response latency and contrast of the $0.5 \mathrm{cpd}$ grating for each observer. In addition, RTs obtained using a single 5.0-cpd

Figure 1. Mean correct reaction times to signal the orientation of 5.0-cpd gratings (triangles) and 0.5-cpd gratings (circles). The contrast of the 5.0-cpd grating was held constant at $30 \%$, whereas the contrast of the 0.5 -cpd grating was varied as indicated along the abscissa. Open symbols denote latencies to simple gratings, and the closed symbols denote latencies to compound gratings. The RTs to the simple 5.0-cpd grating are thus indicated both as a horizontal dashed line and as the open triangle at $0 \%$ contrast for the low-frequency component. RTs to the simple 0.5-cpd grating as a function of contrast are indicated by the dashed curves (and open circles). Each data point includes 100 presentations minus the incorrect responses. 
grating at $30 \%$ contrast are indicated by the horizontal dashed lines and the open triangles. A noteworthy aspect of these data is the substantial disparity in the high- and low-frequency contrast needed to produce a match in RT. Similar effects were first reported by Breitmeyer (1975), who also showed that RTs remain faster for low-frequency gratings even when high- and low-frequency gratings are matched for subjective contrast. The contrasts for the 0.5cpd grating needed to match the 5.0-cpd grating at $30 \%$ contrast are indicated in Table 2. These matches were obtained by the method of adjustment while the observers viewed a bipartite field in which both gratings appeared for $1,000 \mathrm{msec}$. It is clear that the contrast matching data replicate those of Breitmeyer (1975), since in no case did the subjective contrast matches equal those contrasts needed to produce the same RT to the simple high- and low-frequency gratings.

Reactions to the compound gratings. Figure 1 also illustrates RTs to both high- and low-frequency targets when the observers were presented with the compound gratings. Given the large mismatch in contrast needed to match CRTs to the components, the contrast of the 5.0cpd grating was held constant at $30 \%$ while the lowfrequency contrast varied from $0 \%$ to $15 \%$ (indicated on the abscissa). It is clear that the presence of the lowfrequency grating substantially slowed CRTs to a highfrequency target, and that CRTs to low-frequency targets were essentially unaffected by the presence of the highfrequency grating. RTs to high- and low-frequency targets were analyzed in separate analyses of variance (ANOVAs). An ANOVA of the high-frequency CRTs (observers $\times$ low-frequency contrast, including $0 \%$ ) indicated a strong effect of low-frequency contrast $[F(6,18)$ $=19.33, p<.0001]$. This shows that as the contrast of the low-frequency mask is reduced from $15 \%$ to $0 \%$, CRTs to the high-frequency target become faster. Post hoc comparisons (Newman-Keuls) of these data indicated that the presence of the mask significantly interfered with (i.e., slowed) responses to the high-frequency target for all lowfrequency contrasts except the lowest used for each observer (which was $2.5 \%$ for G.S.B. and H.C.H. and $1.25 \%$ for C.P.C. and C.M.R.). CRTs for low-frequency targets were analyzed in a separate two-factor ANOVA (mask/no mask $\times$ low-frequency contrast). This analysis showed that low-frequency contrast had a significant effect $[F(4,12)=10.34, p<.001]$ but that the presence of the high-frequency mask did not effect low-frequency RTs $[F(1,3)=.52]$.

Congruency effects. The effect of mask orientation on RTs to low- and high-frequency targets is illustrated in

Table 2

Contrast of a 0.5-cpd Grating Needed to Match a 5.0-cpd Grating at $30 \%$ Contrast for 4 Observers

\begin{tabular}{lccccr}
\hline \multicolumn{5}{c}{ Grating at 30\% Contrast for 4 Observers } \\
\hline \multicolumn{5}{c}{ Observer } \\
\hline C.M.R. & H.C.H. & G.S.B. & C.P.C. & Mean & SD \\
\hline $25 \%$ & $21 \%$ & $20 \%$ & $15.5 \%$ & 20.4 & 3.9 \\
\hline
\end{tabular}

Note-Each observer's mean is based on five subjective contrast matches.
Figure 2. The ordinate represents the difference (in milliseconds) between RTs when the mask orientation differed from the target orientation (i.e., incongruent compound) versus RTs to compounds in which both components were of the same orientation (congruent compounds). The differences for high-frequency RTs are indicated by triangles; those for low-frequency targets are indicated by circles. It is clear that mask orientation has a substantial effect on responses to the high-frequency target, but that mask orientation has little impact on lowfrequency judgments (i.e., the difference scores cluster around zero). Thus, these data make the same point as Figure 1; the speed of low-frequency orientation judgments is unaffected by the high-frequency grating, but the low-frequency grating clearly interferes with responses to the high-frequency grating. This interference clearly depends on the contrast of the low-frequency mask. All of these points are supported by a three-factor analysis of variance (congruency $\times$ contrast $\times$ target frequency). There was a main effect of low-frequency contrast $[F(1,3)=127.8, p<.001]$ and a main effect of target frequency $[F(1,3)=13.03, p<.04]$. The interaction between congruency and target frequency was significant $[F(1,3)=23.9, p<.02]$, indicating that the congruency effect depends on target frequency. The interaction between low-frequency contrast and target frequency was also significant $[F(4,12)=17.2, p<.0001]$, as was the triple interaction between congruency, contrast, and target $[F(4,12)=7.5, p<.01]$.

Post hoc comparisons indicated a significant effect of congruency on high-frequency targets for all lowfrequency contrasts except the lowest level tested in each observer. In comparison, there were no significant congruency effects for low-frequency targets at any contrast. Additional comparisons indicated that low-frequency RTs to incongruent patterns were faster than high-frequency RTs to congruent patterns at all low-frequency contrasts above $7.5 \%$, indicating that RTs to the low-frequency component are faster than responses to the high-frequency component, even under conditions that favor highfrequency responses.

Finally, additional post hoc comparisons between CRTs to simple high-frequency targets and CRTs to the same grating in the presence of congruent low-frequency masker indicated that significant amounts of interference were generated at low-frequency contrasts above $5.0 \%$ (in C.P.C. and C.M.R.) or 7.5\% (in H.C.H. and G.S.B.). The magnitude of this interference ranges between 60 and $85 \mathrm{msec}$, depending on low-frequency contrast.

\section{Discussion}

These data reveal a clear asymmetry in the suprathreshold processing of high and low spatial frequencies: interference that favors low-frequency processing is obtained even when the contrasts of the components are adjusted so as to produce equivalent CRTs when each component is presented alone. The effects of mask orientation are similarly asymmetric. A similar pattern of 


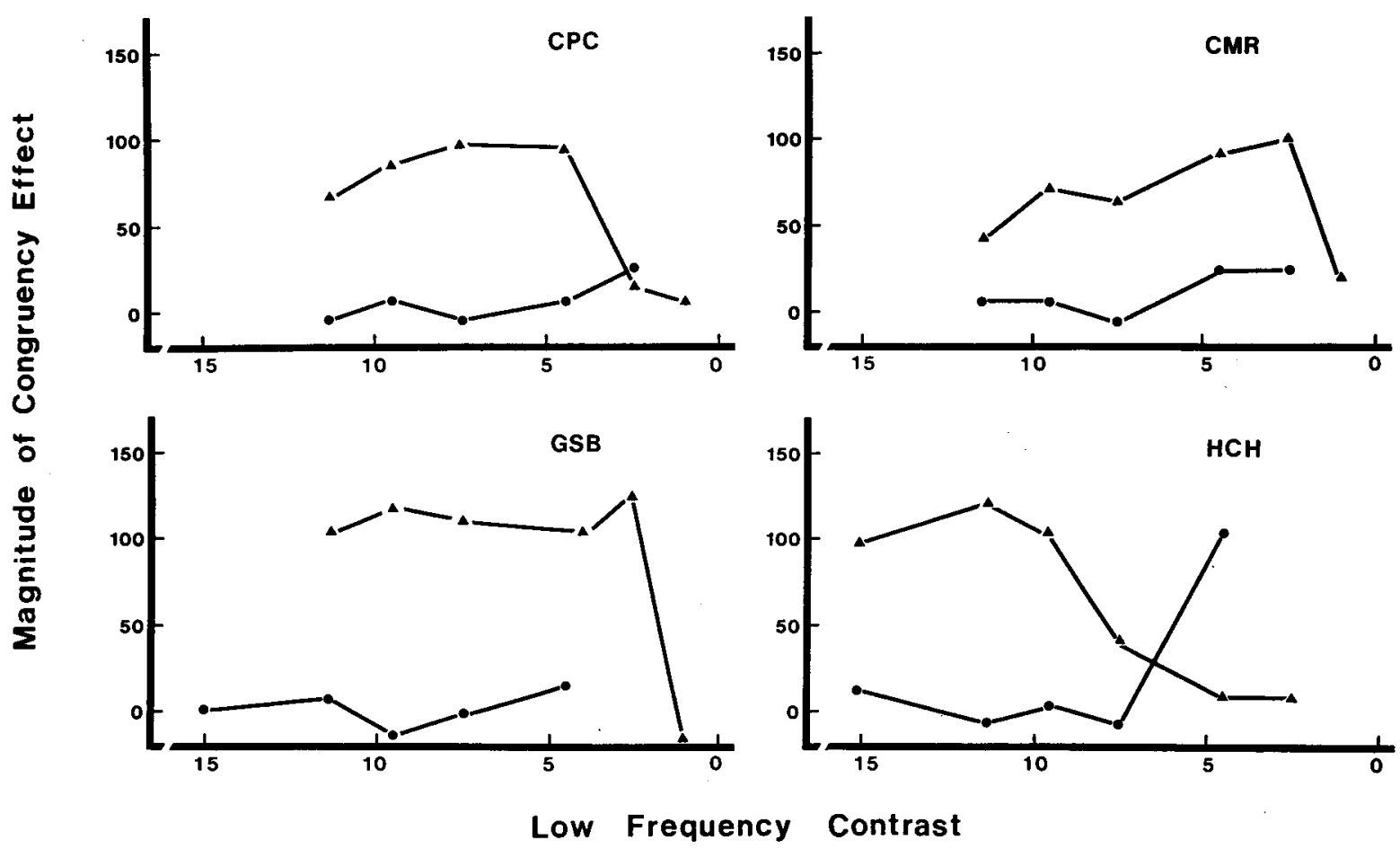

Figure 2. The effect of target-mask congruency on the speed of target-orientation judgments. The magnitude of the congruency effect is simply the difference between RTs to targets when the mask is the opposite versus the same orientation of the target. Triangles represent the data for the 5.0-cpd grating, and circles represent the data for the 0.5-cpd grating. The RTs to the 5.0-cpd gratings clearly depend on the orientation of the 0.5-cpd mask (RTs are slowest when the target and mask are in opposite orientations), but there is no such dependency for the 0.5-cpd targets. In addition, the effect of low-frequency mask orientation diminishes with reductions in low-frequency contrast.

results has been reported by Hughes, Layton, Baird, \& Lester (1984) when using clustered line segments as stimuli.

An interpretation of these data appears to require consideration of two possibly separable effects. First, a lowfrequency masker interferes with CRTs to high-frequency targets, whereas a high-frequency masker produces little or no interference with low-frequency CRTs. Second, the magnitude of this low-frequency interference depends on the orientation of the masker, such that an orthogonal mask generates more interference (both in terms of lengthened CRTs and increased error rates) than a mask in the same orientation as the target.

The fact that mask orientation influences the magnitude of interference immediately suggests that response competition may play some role in generating these effects. There are important aspects of these data which suggest that the effect is not entirely attributable to response competition, however. First, interference occurs on trials in which the low-frequency mask is presented in the same orientation as the high-frequency target. Second, CRTs to low-frequency targets presented with orthogonally oriented high-frequency maskers (where response competition should be operating) are faster than CRTs to highfrequency targets presented with low-frequency maskers of the same orientation (where there should be no response competition). This clearly suggests that some factor other than response competition is involved in the effect, and, in fact, the other factor(s) exert a stronger influence on the overall RTs than does the influence of response competition (since response times to high-frequency targets are slower even when response competition should favor the high-frequency components of the compound).

Finally, the fact that response competition (i.e., the congruency between target and mask) is found only for highfrequency targets also suggests a source of interference that occurs prior to a (presumably) later stage of response competition. That is, if the sensory information concerning each grating is accumulated at the same rate and orthogonal gratings produce competing response tendencies which slow RTs, then one should see congruency effects for low- as well as high-frequency targets. The data can therefore be taken to suggest that, even though RTs to the two components are equated, some aspect of the internal response to the low frequency is greater than that for the high frequency when the gratings are presented together. According to this view, response competition has an effect, but its influence depends on the rate at which earlier information about the presence of each component grating is accumulating. If these two rates differ, then one might expect to see more response competition (a greater congruency effect) when the slower sensory channel en- 
codes the target grating. These considerations lead to the possibility that, when the two gratings are presented in compound, the internal responses to each component interact in a way that favors the response to the lower frequency.

One possible mechanism that would produce such an effect is asymmetric inhibitory interactions between channels tuned to the high- and low-frequency components of these compound gratings. Interchannel inhibition has been previously suggested on the basis of adaptation studies (DeValois, 1977; Tolhurst, 1972; Tolhurst \& Barfield, 1978), and could relate to the detection and recognition data of Nachmias and Weber (1975). The possibility that transient channels inhibit sustained channels has been suggested by several electrophysiological studies (see Lennie, 1980, for a review) and psychophysical studies (e.g., Breitmeyer \& Ganz, 1976; Breitmeyer et al., 1980). Moreover, there is evidence that the presence of gratings oriented orthogonally to the preferred orientations of cortical cells can inhibit the cell's response to optimal stimuli (Morrone et al., 1982; Petrov et al., 1980). Morrone et al. (1982) report that this cross-orientation inhibition is stronger in simple cells than in complex cells, and there is evidence that simple cells receive their input primarily from $\mathrm{x}$ cells (sustained cells) whereas complex cells received their input primarily from y cells (transient cells; e.g., Lennie, 1980; Stone, Dreher, \& Leventhal, 1979). In view of the evidence that transient channels respond best to low spatial frequencies while sustained channels respond best to high spatial frequencies (e.g., Breitmeyer, 1975; Burbeck \& Kelly, 1981; Harwerth \& Levi, 1978; Tolhurst, 1975a, 1975b), it might be suggested that the asymmetric interference observed in the experiment could be attributed to inhibition of sustained channels by transient channels. If this were the case, removing the temporal transient in the onset of these patterns should reduce the magnitude of the low-frequency interference effect on high-frequency orientation judgments, since the lack of an abrupt onset should confer a processing advantage to the high-frequency grating. This possibility was explored in Experiment 2.

\section{EXPERIMENT 2}

Using a reaction time task, Tolhurst (1975a) demonstrated the differential temporal sensitivity of channels tuned to high and low spatial frequencies. He showed that RTs to low-frequency gratings at near threshold contrast tend to occur just after any sudden transient in the stimulus (including the offset). In comparison, RTs to highfrequency gratings tend to occur in a unimodal distribution following the pattern onset. In the second experiment, I sought to take advantage of this temporal dependency to determine whether the magnitude of low-frequency interference varies with the temporal parameters of the compound gratings. Thus, the difference between this and the previous experiment is that the stimulus onset was gradual rather than abrupt. If the interference found in Experi- ment 1 somehow reflected interactions between transient and sustained channels, then gradual onsets should reduce the magnitude of the interference by conferring an advantage to the higher frequency component.

\section{Method}

Observers. The same 4 observers who served in Experiment 1 participated in this experiment.

Stimuli and Procedure. The stimuli and procedures were the same as in Experiment 1, the only difference being in the time course of the pattern onsets. In this experiment, the stimuli took $500 \mathrm{msec}$ to reach their terminal contrast, and remained at that contrast for an additional $100 \mathrm{msec}$. The screen was blanked when the observer responded or when the 600 -msec duration expired, whichever came first. The change in contrast was linear throughout the $500-\mathrm{msec}$ onset period.

\section{Results}

The RT data are illustrated in Figure 3. These data are presented in the same format as Figure 1. There are several noteworthy aspects in these data. First, RTs obtained under the gradual onset condition are consistently slower than those obtained in Experiment 1 under comparable contrasts. This is almost certainly attributable to the slow rate of change in contrast as the RT clock began at the beginning of the onset ramps. Second, lowfrequency contrasts, which produced substantial interference in the first experiment, produced less interference under conditions of gradual onset. Third, the terminal lowfrequency contrast needed to match RTs to the highfrequency grating were elevated relative to the contrasts that produced equivalent RTs in Experiment 1.

Figure 4 illustrates the magnitude of low-frequency interference on high-frequency RTs in the first and second experiments. In this figure, the magnitude of interference is defined as the difference between RTs to high-frequency targets presented in compound and those to the highfrequency gratings presented alone. It is clear that the magnitude of low-frequency interference depends on the onset condition; gradual onsets produce less interference than does an instantaneous onset. An ANOVA on these data (low-frequency contrast $X$ onset condition) indicated a significant effect of low-frequency contrast $[F(3,9)=$ $11.9, p<.001]$ and onset condition $[F(1,3)=10.6$, $p<.05]$, but the interaction was not significant $[F(4,9)$ $=1.8, p=.22]$. Low-frequency contrast accounted for $18.5 \%$ of the total variance, whereas the temporal onset accounted for $37.7 \%$ of the total variance. An analysis of RTs to low-frequency targets again indicated no significant interference from the high-frequency mask.

\section{Discussion}

The data from Experiment 2 indicate that a procedure that differentially influences low- relative to high-spatialfrequency mechanisms attenuates the ability of the lowfrequency grating to interfere with responses to the highfrequency target. It would appear that, under these conditions, the interference between these stimuli is intimately related to the responses of those sensory channels thought 

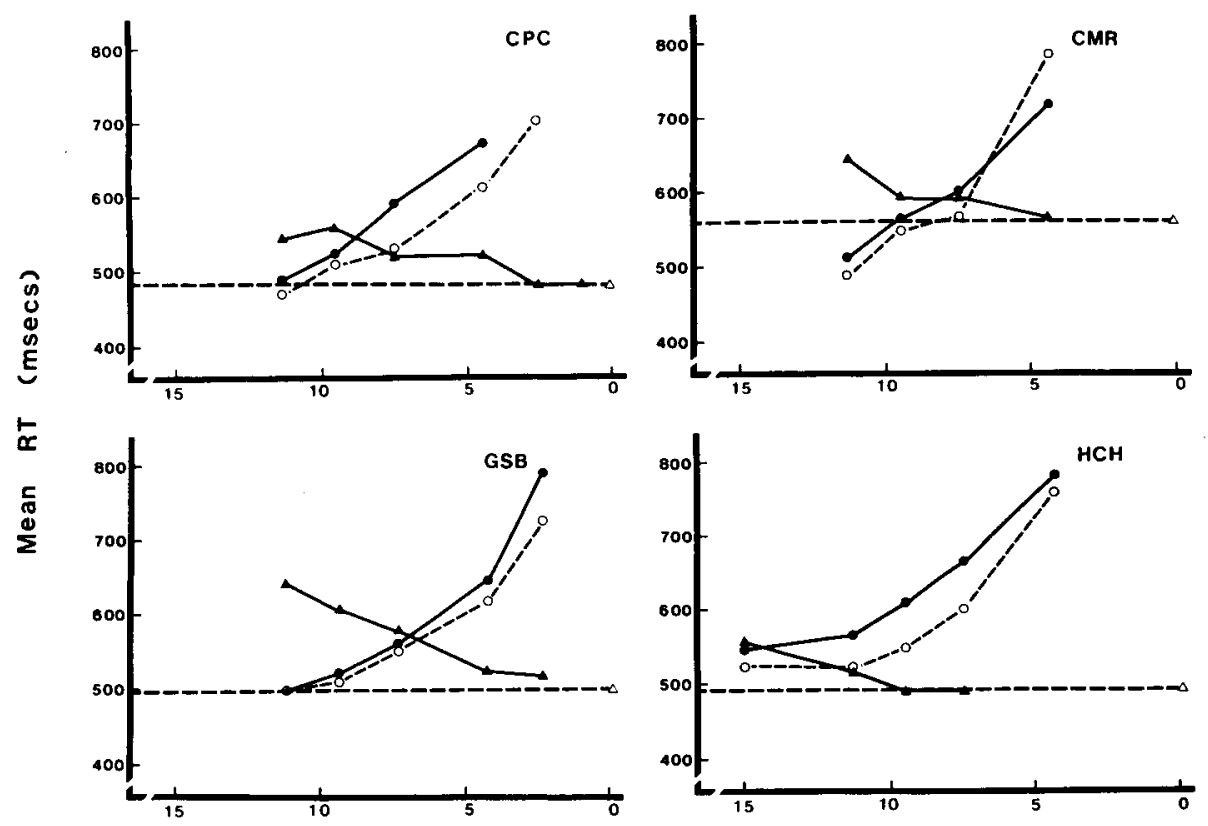

Low Frequency Contrast

Figure 3. Data similar to that illustrated in Figure 1, except that the stimulus onset was gradual (linear increase over $500 \mathrm{msec}$ ) instead of abrupt. Notice that the scale of the ordinate has changed, but the figure is otherwise the same as Figure 1.

to encode them. The implication is that the interference in RTs reflects interference between sustained and transient mechanisms. The third experiment examined this possibility from another point of view by looking at the interactions between redundant high- and low-frequency signals as revealed by the speed of orientation judgments.

\section{EXPERIMENT 3}

The outcomes of the first two experiments are consistent with the view that the RT measurements are closely related to the activity of (presumably cortical) neurons known to respond to the same patterns. In addition, the physiological studies of Morrone et al. (1982) suggest that "cross-orientation" inhibition might relate to the observation of asymmetric interactions between high- and lowfrequency components of these compound gratings. It is important to recognize that an interpretation of these interference effects in terms of cross-orientation inhibition need not imply that the inhibition is restricted to orthogonally oriented gratings, or that it is more potent with orthogonal gratings. Rather, it implies only that the inhibition is broadly tuned with respect to orientation (see Morrone et al., 1982) and therefore may still be present with the orthogonal gratings. According to this view, then, the interference is present regardless of mask orientation, but is enhanced with orthogonal masks because of the additional influence of response competition (see Discussion of Experiment 1).
In Experiment 3, an attempt was made to measure the manner in which these high- and low-frequency stimuli interact in producing speeded orientation judgments when the components present redundant orientation cues. The approach was to compare RTs to simple high- and lowfrequency gratings with those obtained using compound gratings whose components were always in the same orientation (which varied randomly across trials). If the components of the compounds were detected by independent parallel channels, then RTs to the compounds should be faster than RTs to either component presented alone by an amount predictable by probability summation between the distribution of RTs generated by the simple gratings. Any inhibitory interactions between the stimuli should produce RTs to the compound gratings that were slower than those expected on the basis of probability summation between independent channels tuned to the frequency of each component.

\section{Method}

Observers. The same 4 observers participated in Experiment 3. Stimuli and Procedure. The stimuli and procedure were the same as in Experiment 1, except that the components in the compound gratings were always in the same orientation (either horizontal or vertical, which varied randomly from trial to trial).

\section{Results}

The results are presented in Figure 5, which indicates the mean RT for correct responses for simple high- and low-frequency gratings and for the (orientationally) redun- 


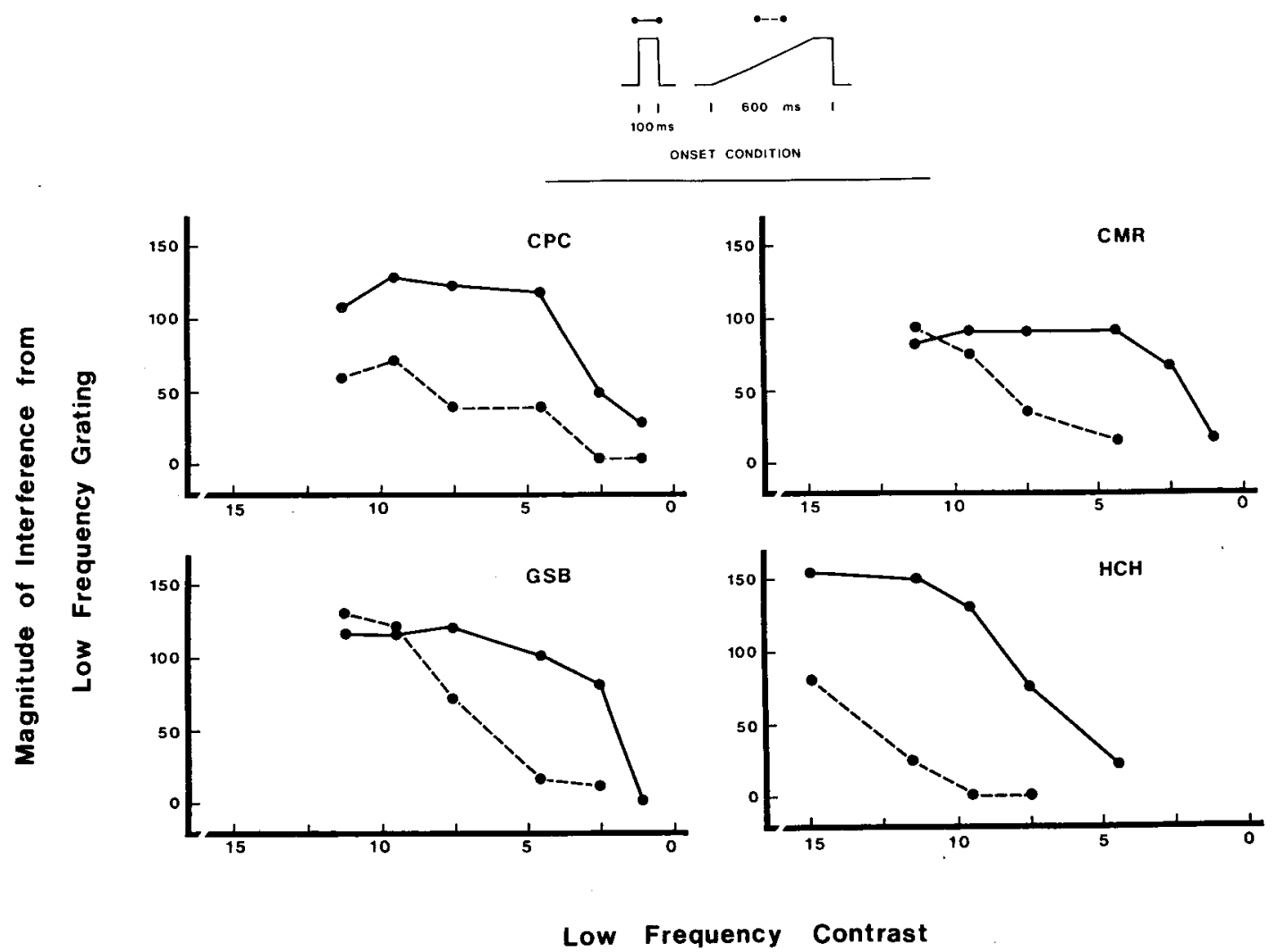

Figure 4. The magnitude of interference generated by the 0.5 -cpd sinusoid on the speed of judging the orientation of the 5.0-cpd sinusoid. The ordinate indicates the difference between RTs to the 5.0-cpd grating presented in compound with the 0.5-cpd mask and RTs to the 5.0-cpd grating presented alone. The abscissa represents the contrast of the 0.5-cpd mask. The solid lines indicate the low-frequency interference with an abrupt onset, and the dotted lines indicate interference under the gradual onset condition. There was no significant interference by the 5.0-cpd grating in either onset condition.

dant compounds. As can be seen in the figure, CRTs to the simple gratings are very similar, a result of the fact that the contrast of each grating was adjusted so as to produce similar RT distributions. Also included in Figure 5 is the level of performance predicted on the basis of suprathreshold probability summation. The estimates were derived from the RT distributions obtained from each component when presented alone; the exact procedure is described below. It is clear from Figure 5 that the observers derived little benefit from the redundancy of the compounds. An ANOVA of these data (average RT for each observer $\times 3$ types of pattern) showed no significant difference among the three pattern conditions. To achieve more statistical power, the individual RTs from each observer were analyzed separately. Two of the observers showed a significant redundancy gain according to the latter analysis (H.C.H. and C.P.C.); the other two did not.

Estimates of probability summation. Blake, Martens, and DiGianfilippo (1980) and Blake, Martens, Garrett, and Westendorf (1980) have provided an empirical analysis which provides a method for estimating probability summation from suprathreshold RT data. The same method was used by Klein (1977). Although Blake's anal- ysis was concerned with binocular summation, the method should work for summation effects between any two independent sensory channels (see, e.g., Klein, 1977). The data in Figure 5 are compared with a simulation of probabilistic summation of response times using the following procedure. RTs are randomly selected from the RT distribution obtained from the high- and low-frequency gratings. The faster of the two selected RTs is taken as an estimate of the RT to the compound on the assumption that when viewing orientationally redundant compounds, the first component recognized is sufficient to generate an orientation judgment. Iterations of this procedure generate a distribution of simulated RTs to the compound, and the mean of this distribution is taken as an estimate of the mean RT to compounds based on a simple "horse-race" model of response generation. Unpublished work in this laboratory using binocular summation of RTs has indicated that the obtained binocular benefit exceeds the estimate of probability summation using this procedure on $70 \%$ of the comparisons (White, 1984). This figure corresponds closely to that reported by Blake, Martens, and DiGianfilippo (1980). In contrast, the improvement in RT to the orientationally redundant compound 

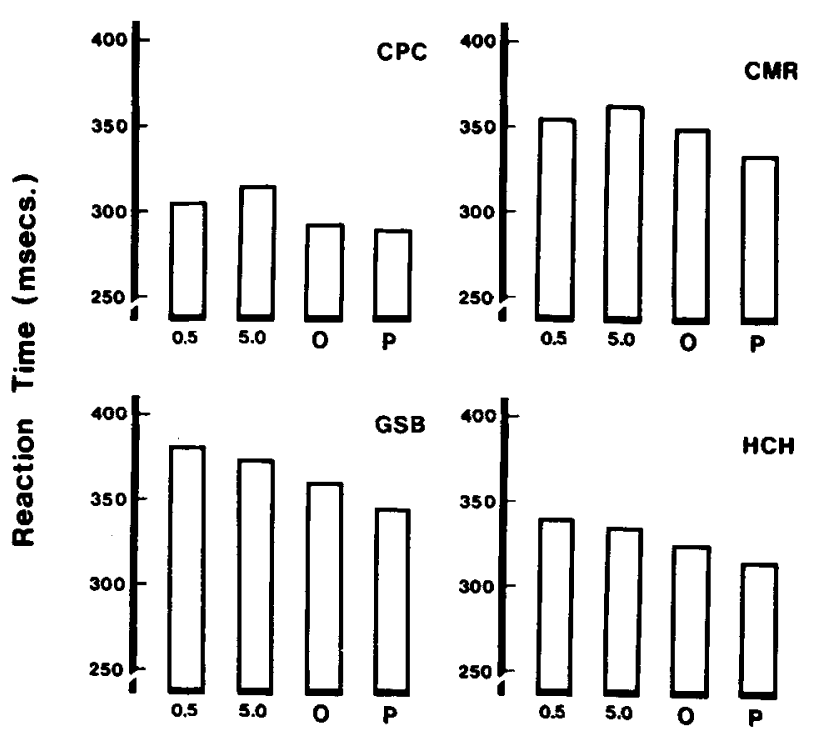

Stimulus Condition

Figure 5. Average latencies to judge the orientation of single gratings ( 0.5 and 5.0 cpd) and compound gratings $(O)$ in which both components were always in the same orientation. The " $O$ " denotes obtained RTs to the orientationally redundant compounds, and the "P" denotes the predicted RTs obtained from a simple model of suprathreshold probability summation, as described in the text.

gratings in this experiment failed to exceed the estimate due to independent summation in all 4 observers (although there was one virtual tie, C.P.C.). Three of the observers fell about midway between no benefit and that expected according to the horse-race model.

\section{GENERAL DISCUSSION}

The pattern of results obtained in this investigation is consistent with the suggestion that activity in transient channels inhibits activity in sustained channels (Breitmeyer, 1978; Breitmeyer \& Ganz, 1976; Valberg \& Breitmeyer, 1980). However, if these data do reflect a masking effect, it differs from that found in previous masking experiments in the direction of the asymmetry: that is, masking effects on grating detection have tended to reveal asymmetries that favor high rather than low spatial frequencies (Nachmias \& Weber, 1975; Stromeyer \& Klein, 1974; see, however, Mezrich, 1978).

One potentially important difference between the present results and those of masking studies that have examined detection thresholds is that grating detection might be mediated by changes in the appearance of the masker rather than identification of the test grating per se. Nachmias and Weber (1975) and Stromeyer and Klein (1974) have both indicated that the subjective reports of their observers suggest this possibility. In contrast, the present experiments require that the observer specifically identify the orientation and the spatial frequency of the target.
Another important difference between the present experiments and those of Nachmias and Weber (1975) and Stromeyer and Klein (1974) is in the frequency of the gratings: Nachmias and Weber (1975) and Stromeyer and Klein (1974) used frequencies of 3.0 and $9.0 \mathrm{cpd}$, whereas I used 0.5 and $5.0 \mathrm{cpd}$. If there are inhibitory interactions between transient and sustained channels, it is unlikely that they could be observed using 3.0- and 9.0-cpd gratings, since the transient mechanisms are generally thought to respond poorly to frequencies above $3.0 \mathrm{cps}$ (Burbeck \& Kelly, 1981; Harwerth \& Levi, 1978; King-Smith \& Kulikowski, 1975; Legge, 1978; Tolhurst; 1975a, 1975b).

However, the present data are very similar to data previously reported by Hughes et al. (1984) on asymmetric interference between high- and low-resolution pattern cues. Thus, these data represent an important extension of a general finding that low-resolution cues tend to dominate visual pattern processing in reaction time tasks (e.g., Boer \& Keuss, 1982; Hughes et al., 1984; Kinchla \& Wolf, 1979; Navon, 1977; Paquet \& Merikle, 1984). It seems possible that processes such as image segmentation, form perception, and the selection of targets for foveation during inspection of scenes may derive benefits from this lowfrequency dominance.

\section{REFERENCES}

Blake, R., Martens, B., DiGianfilippo, A. (1980). Reaction time as a measure of binocular interaction in human vision. Investigative Ophthalmology, 19, 930-941.

Blake, R., Martens, B., Garrett, A., \& Westendorf, D. (1980). Estimating probability summation for binocular reaction time data. Perception \& Psychophysics, 27, 375-378.

Blakemore, C., \& Campbell, F. W. (1969). On the existence of neurons in the human visual system selectively sensitive to the orientation and size of retinal images. Journal of Physiology (London), 203, 237-260.

BoER, L. C., \& Keuss, P. J. G. (1982). Global precedence as a postperceptual effect: An analysis of speed-accuracy tradeoff functions. Perception \& Psychophysics, 31, 358-366.

BREITMEYER, B. G. (1975). Simple reaction time as a measure of the temporal response properties of transient and sustained channels. $V i$ sion Research, 15, 1411-1412.

BREITMEYER, B. G. (1978). Metacontrast with black and white stimuli: Evidence for inhibition of on- and off-sustained activity by either onor off-transient activity. Vision Research, 18, 1443-1448.

BREITMEYER, B. G., \& GANZ, L. (1976). Implications of sustained and transient channels for theories of visual pattern masking, saccadic suppression, and information processing. Psychological Review, 83, 1-36.

Breitmeyer, B. G., ValberG, A., Kertenbach, W., \& Neumeyer, C. (1980). The lateral effect of oscillation of peripheral luminance gratings on the foveal increment threshold. Vision Research, 20, 799-805.

Burbeck, C. A., \& KelLy, D. H. (1981). Contrast gain measurements and the transient/sustained dichotomy. Journal of the Optical Society of America, 71, 1335-1342.

CAmprell, F. W., \& Robson, J. G. (1968). Application of Fourier analysis to the visibility of gratings. Joumal of Physiology (London), 197, $551-566$.

DEVALOIS, K. (1977). Spatial frequency adaptation can enhance contrast sensitivity. Vision Research, 17, 1057-1065.

Dubin, M. W., \& Cleland, B. G. (1977). Organization of visual inputs to interneurons of lateral geniculate nucleus of the cat. Journal of Neurophysiology, 40, 410-427. 
Graham, N., \& Nachmias, J. (1971). Detection of grating patterns containing two spatial frequencies: A comparison of single channel and multiple-channels models. Vision Research, 11, 251-259.

HARWERTH, R. S., \& LEVI, D. M. (1978). Reaction time as a measure of suprathreshold grating detection. Vision Research, 18, 1579-1586.

Henning, G. B., Hertz, B. G., \& Broadbent, D. E. (1975). Some experiments bearing on the hypothesis that the visual system analyses spatial patterns in independent bands of spatial frequency. Vision Research, 15, 887-897.

Hoffmann, K.-P., Stone, J., \& Sherman, S. M. (1972). Relay of receptive-field properties in dorsal lateral geniculate nucleus of the cat. Joumal of Neurophysiology, 35, 518-531.

Hughes, H. C., LAYTon, W. M., Baird, J. C., \& Lester, L. S. (1984). Global precedence in visual pattern recognition. Perception \& Psychophysics, 35, 361-371.

Kelly, D. H., \& BuRBECK, C. A. (1984). Critical problems in spatial vision. CRC Critical Reviews in Biomedical Engineering, 10, 125-174.

KINCHLA, R. A., \& Wolf, J. M. (1979). The order of visual processing: "Top-down," "bottom-up," or "middle-out." Perception \& Psychophysics, 25, 225-231.

KING-Smith, P. E., \& Kulikowski, J. J. (1975). The detection of gratings by independent activation of line detectors. Journal of Physiology (London), 247, 237-271.

KLEIN, R. M. (1977). Attention and visual dominance: A chronometric analysis. Journal of Experimental Psychology: Human Perception \& Performance, 3, 365-378.

LEGGE, G. E. (1978). Sustained and transient mechanisms in human vision: Temporal and spatial properties. Vision Research, 18, 69-81.

LENNIE, P. (1980). Parallel visual pathways: A review, Vision Research, 20, 561-594.

Levick, W. R., Cleland, B. G., \& Dubin, M. W. (1972). Lateral geniculate neurons of cat: Retinal inputs and physiology. Investigative Ophthalmology, 11, 302-311.

MEZRICH, J. J. (1978). Modification of spatial frequency processing rates with multiple frequency stimuli. Vision Research, 18, 1505-1507.

Morrone, M. C., BurR, D. C., \& MAFFE, L. (1982). Functional implications of cross-orientation inhibition of cortical visual cells. I. Neurophysiological evidence. Proceedings of the Royal Society, London, Series B, 216, 335-354.

Nachmias, J., SANSBury, R., Vasilev, A., \& Weber, A. (1973). Adaptation to square-wave gratings: In search of the elusive third harmonic. Vision Research, 13, 1335-1342.

NACHMIAS, J., \& WEBER, A. (1975). Discrimination of simple and complex gratings. Vision Research, 15, 217-223.

Navon, D. (1977). Forest before the trees: The precedence of global features in visual perception. Cognitive Psychology, 9, 353-383.

NoDA, H. (1975). Depression of excitability of relay cells of lateral geniculate nucleus following saccadic eye movements in the cat. Journal of Physiology (London), 249, 87-102.
Pantle, A., \& Sekuler, R. (1968). Size-detecting mechanisms in human vision. Science, 162, 1146-1148.

Paquet, L., \& Merikle, P. M. (1984). Global precedence: The effect of exposure duration. Canadian Journal of Psychology, 38, 45-53.

Petrov, A. P., Pigarev, I. N., \& Zenkin, G. M. (1980). Some evidence against Fourier analysis as a function of the receptive fields in cat's striate cortex. Vision Research, 20, 1023-1025.

SaChS, M. B., NAchmias, J., \& Robson, J. G. (1971). Spatial-frequency channels in human vision. Journal of the Optical Society of America, 61, 1171-1186.

SINGER, W., \& BEDWORTH, N. (1973). Inhibitory interactions between $\mathrm{X}$ and $\mathrm{Y}$ units in the cat lateral geniculate nucleus. Brain Research, 49, 291-307.

Singer, W., Poppel, E., \& Creutzfeldt, O. (1972). Inhibitory interaction in the cat's lateral geniculate nucleus. Experimental Brain Research, 14, 210-226.

Stone, J., Dreher, B., \& Leventhal, A. (1979). Hierarchical and parallel mechanisms in the organization of visual cortex. Brain Research Reviews, 1, 345-394.

Stromeyer, C. F., III, \& Julesz, B. (1972). Spatial-frequency masking in vision: Critical bands and spread of masking. Journal of the Optical Society of America, 62, 1221-1232.

Stromeyer, C. F., III, \& KLEIN, S. (1974). Spatial frequency channels in human vision as asymmetric (edge) mechanisms. Vision Research, 14, 1409-1420.

ToLhURST, D. J. (1972). Adaptation to square-wave gratings: Inhibition between spatial-frequency channels in the human visual system. Journal of Physiology (London), 226, 231-249.

ToLHURST, D. J. (1973). Separate channels for the analysis of the shape and the movement of a moving visual stimulus. Journal of Physiology (London), 231, 385-402.

TOLHURST, D. J. (1975a). Reaction times in the detection of gratings by human observers: A probabilistic mechanism. Vision Research, 15, 1143-1149.

TolhuRst, D. J. (1975b). Sustained and transient channels in human vision. Vision Research, 15, 1151-1155.

TOLHURST, D. J., \& BARFIELD, L. P. (1978). Interactions between spatial frequency channels. Vision Research, 18, 951-958.

VALBERG, A., \& BREITMEYER, B. G. (1980). The lateral effect of oscillation of peripheral luminance gratings: Test of various hypotheses. Vision Research, 20, 789-798.

WHITE, T. (1984). Effects of flash luminance and flash eccentricity on binocular summation: Reaction time measures. Unpublished honors thesis, Department of Psychology, Dartmouth College.

(Manuscript received October 30, 1985; revision accepted for publication July 9,1986 .) 